\title{
AN INEQUALITY FOR MEANS WITH APPLICATIONS
}

\author{
JAN-CHRISTOPH SCHLAGE-PUCHTA
}

\begin{abstract}
We show that an almost trivial inequality between the first and second moment and the maximal value of a random variable can be used to slightly improve deep theorems.
\end{abstract}

One often estimates individual values of a function by computing a certain means. This approach is particularly useful in situations where the conjectured maximum of a function is close to its mean, as is often the case in number theory. Here, we give a simple method which sometimes allows to improve the resulting estimates. To ease aplications, we formulate our result in the language of probability theory.

Theorem 1. Let $\xi$ be a non-negative real random variable, and suppose that $\mathbf{E} \xi=$ 1 , and $\mathbf{E} \xi^{2}=a$ with $a>1$. Then the probability $P(\xi \geq a)$ is positive, and for every $b<a$ we have

$$
\int_{|\xi|>b} \xi^{2} \geq a-b .
$$

Proof. We have

$$
\int_{|\xi| \leq b} \xi^{2} \leq b \int_{|\xi|>b} \xi \leq b \int \xi=b,
$$

which implies the claimed inequality. Now suppose that $|\xi|<a$ almost surely. Then there exists some $\epsilon>0$, such that $\int_{|\xi| \leq a-\epsilon} \xi \geq \frac{1}{2}$, and we obtain

$$
\begin{aligned}
a=\int \xi^{2}=\int_{|\xi| \leq a-\epsilon} \xi^{2}+\int_{|\xi|>a-\epsilon} & \xi^{2} \\
\leq & (a-\epsilon) \int_{|\xi| \leq a-\epsilon} \xi+a \int_{|\xi|>a-\epsilon} \xi \leq \frac{(a-\epsilon)+a}{2}<a,
\end{aligned}
$$

a contradiction.

We now give three applications to quite different areas.

Our first application shows that the fourth moment of the Riemann $\zeta$-function is dominated by large values of $\zeta$, in fact, by values which are so large that the fourth moment itself cannot guarantee them to exist.

Date: Novembre 25, 2006.

1991 Mathematics Subject Classification. Primary 26D15; Secondary 11M06, 15A15, 20C30.

Key words and phrases. inequality, mean values, fourth mean of Zeta-function, Hadamardmatrices, Character degrees. 
Corollary 1. There is a constant $C$ such that for $t>T_{0}(\epsilon)$ and $H>T^{2 / 3} \log ^{C} T$ we have

$$
\int_{\left\{t \in[T, T+H]:\left|\zeta\left(\frac{1}{2}+i t\right)\right|>\frac{1}{4 \pi^{2}} \log ^{3 / 2} t\right\}}\left|\zeta\left(\frac{1}{2}+i t\right)\right|^{4} d t \geq \frac{1-\epsilon}{4 \pi^{2}} T \log ^{4} T+\mathcal{O}\left(T \log ^{3} T\right) .
$$

Proof. Ingham proved

$$
\left.\int_{0}^{T}\left|\zeta\left(\frac{1}{2}+i t\right)\right|^{2} d t=T \log \frac{T}{2 \pi}+(2 \gamma-1) T+\mathcal{O} / T^{1 / 2+\epsilon}\right)
$$

and Ivic and Motohashi[2] showed that

$$
\int_{0}^{T}\left|\zeta\left(\frac{1}{2}+i t\right)\right|^{4} d t=T \cdot P(\log T)+\mathcal{O}\left(T^{2 / 3} \log ^{C} T\right),
$$

where $P$ is a polynomial of degree 4 with leading term $\frac{1}{2 \pi^{2}}$ (confer also [1]). Now apply Theorem 1 by setting

$$
\xi=H \cdot\left|\zeta\left(\frac{1}{2}+i t\right)\right|^{2}\left(\int_{T}^{T+H}\left|\zeta\left(\frac{1}{2}+i t\right)\right|^{2} d t\right)^{-1}
$$

for $t \in[T, T+H]$ chosen at random and $b=\frac{1}{4 \pi^{2}} \log ^{2} T$.

Our second application slightly improves on the approach of Szekeres and Turán [5] on the problem of Hadamard-matrices.

Corollary 2. For every $\epsilon>0$ and $n>n_{0}(\epsilon)$ there exists a skew-symmetric $n \times n$ matrix $A$ with entries \pm 1 satisfying

$$
|\operatorname{det} A|>\left(\frac{n}{64 \pi e^{5}}\right)^{1 / 4} e^{\sqrt{n} \sqrt{n !}}
$$

Proof. Let $A$ be a random skew-symmetric $n \times n$-matrix with entries \pm 1 , and let $s_{k}(n)$ be the $k$-th mean of the determinant of $A$. Szekeres [4] showed that

$$
\begin{aligned}
& s_{1}(n) \sim \frac{1}{\sqrt[4]{8 \pi e n}} e^{\sqrt{n}} \sqrt{n !}, \\
& s_{2}(n) \sim \frac{1}{\sqrt{32 \pi e^{3}}} e^{2 \sqrt{n}} \sqrt{n !} .
\end{aligned}
$$

Our claim now follows by applying our theorem to $\frac{\operatorname{det} A}{s_{1}(n)}$.

Our last result improves on the work of Kerov and Vershik [3] concerning the largest degree of an irreducible character of the symmetric group.

Corollary 3. Let $\epsilon>0$ be given. Then for every $n>n_{0}(\epsilon)$ there exists an irreducible character $\chi$ of $S_{n}$ with $\chi(1)>(1-\epsilon) e^{1 / 4} \sqrt{\pi n} e^{-\sqrt{n}} \sqrt{n !}$.

Proof. All irreducible complex representations of $S_{n}$ can be realized over $\mathbb{R}$, thus

$$
\sum_{\chi} \chi(1)=\#\left\{\pi \in S_{n}: \pi^{2}=\mathrm{id}\right\} \sim \frac{e^{\sqrt{n}-\frac{1}{4}}}{2 \sqrt{\pi n}} \sqrt{n !},
$$


whereas the orthogonality relation implies $\sum_{\chi} \chi(1)^{2}=n$ !. Finally, the number of irreducible characters equals the number $p(n)$ of partitions of $n$, for which we have the asymptotic formula

$$
p(n) \sim \frac{1}{4 n \sqrt{3}} e^{\pi \sqrt{2 n / 3}} .
$$

Define a random variable $\xi$ as $\frac{\chi(1)}{\sqrt{n !}}$, where $\chi$ is chosen at random among all irreducible characters, where each character has the same probability. Then we obtain

$$
\begin{aligned}
\mathbf{E} \xi & \sim \frac{2 \sqrt{3 n}}{e^{1 / 4} \sqrt{\pi}} \exp ((1-\pi \sqrt{2 / 3}) \sqrt{n}) \\
\mathbf{E} \xi^{2} & \sim 4 n \sqrt{3} \exp (-\pi \sqrt{2 n / 3}),
\end{aligned}
$$

and our claim follows.

\section{REFERENCES}

[1] D. R. Heath-Brown, The fourth power moment of the Riemann zeta function, Proc. London Math. Soc. (3) 38 (1979), 385-422.

[2] A. Ivić, Y. Motohashi, On the fourth power moment of the Riemann zeta-function, J. Number Theory 51 (1995), 16-45.

[3] S. V. Kerov, A. M. Vershik, Asymptotic of the largest and the typical dimensions of irreducible representations of a symmetric group, Funktsional. Anal. i Prilozhen. 19 (1985), 25-36; translated in Functional Analysis and Its Applications 19 (1985), 21-31.

[4] G. Szekeres, Determinants of skew type \pm 1 matrices, Period. Math. Hungar. 3 (1973), 229-234.

[5] G. Szekeres, P. Turán, An extremal problem in the theory of determinants, Mat. Termeszett. Ertes. 56 (1937), 796-806; translated in Colected Papers of Paul Turán, P. Erdős ed., Akadémiai Kiadó, Budapest, 1990, 81-87

Mathematisches Institut, Eckerstr. 1, 79104 Freiburg, Germany

E-mail address: jcp@math.uni-freiburg.de 